\title{
Análise automatizada de exames de urina utilizando imagens digitais de dipsticks
}

\author{
Rafaela Barbirato Ferreira ${ }^{1}$, Lucas Ferrari de Oliveira $^{1,2}$ \\ ${ }^{1}$ Curso Bacharelado em Informática Biomédica \\ Universidade Federal do Paraná (UFPR) \\ Curitiba - PR, Brasil \\ ${ }^{2}$ Laboratório de Visão, Robótica e Imagem (VRI) \\ Programa de Pós-Graduação em Informática (PPGInf) \\ Universidade Federal do Paraná (UFPR) \\ Curitiba - PR, Brasil \\ rafaelabarbirato@gmail.com, lferrari@inf.ufpr.br
}

\begin{abstract}
This paper describes a study on the dipsticks analysis for urine tests. For the purposes of this work, a database of dipsticks images was elaborated to enable the development and the performance evaluation of the information extraction techniques proposed. The information extraction was done in HSV and RGB color model. For the tests, the classifier MLP (Multi-layer Perceptron) was selected. The number of layers, neurons, learning rate, momentum and epoch were varied, in order to evaluate the best approach to the classification problem. The algorithm obtained a mean of $91.21 \%$ of success rate, proving to be efficient in the data classification. Experiments have confirmed that the technique is viable.
\end{abstract}

Resumo. Este artigo propõe um estudo sobre a análise de fitas reagentes (dipsticks) para exames de urina. Uma base de imagens de dispsticks foi criada, possibilitando o desenvolvimento e avaliação das técnicas de extração propostas. A extração de características das imagens foi feita utilizando-se os modelos de cores HSV e RGB. Para os testes o classificador escolhido foi o MLP (Multilayer Perceptron). A quantidade de camadas da rede, número de neurônios, taxa de aprendizado, momentos e épocas foram variados, visando melhores resultados de classificação. O MLP obteve uma média de $91,21 \%$ de taxa da acerto, mostrando-se eficiente na classificação dos dados. Os experimentos desenvolvidos confirmaram que a técnica é viável.

\section{Introdução}

No início da medicina o exercício da profissão ficava restrito apenas a observação e exame físico do paciente. Já os estudos laboratoriais apenas podiam ser realizados através das substâncias eliminadas pelo paciente, dentre elas a urina. Acredita-se que um dos procedimentos laboratoriais mais antigos, que tem sido utilizado desde o século XIX para diagnóstico de doenças, é a análise da urina [Strasinger 2000, Amorim et al. 2009].

A análise da urina, ou conhecida também como uroanálise, foi o grande começo da medicina laboratorial. Essa análise da urina era feita por meio de observações básicas, mas que traziam informações diagnósticas como a cor, turvação, odor e até mesmo 
a presença de açúcar em certas amostras, observada pela aproximação de formigas e demais insetos [Bolodeoku and Donaldson 1996, Strasinger and Torquettitoloi 1996]. A uroanálise foi definida pela Associação Brasileira de Normas Técnicas (ABNT, 2005, p.1) como "exame realizado numa amostra de urina humana para determinar os caracteres físicos e químicos e para verificar a presença de elementos figurados ou de outra origem"[ABNT 2005]. Esse exame é o terceiro mais realizado em laboratórios clínicos e pode ser obtido sem nenhum procedimento invasivo. O mesmo fornece informações importantes, tanto no que diz respeito ao diagnóstico e monitoramento de doenças renais ou do trato urinário, quanto para a detecção de doenças sistêmicas e metabólicas que podem estar relacionadas não apenas com os rins [Chien et al. 2007, Strasinger 2000]. Ao longo dos anos, o processo de análise do exame de urina evoluiu, com a finalidade de se obter resultados mais confiáveis e precisos. As técnicas desenvolvidas não envolvem apenas o exame físico, mas também o estudo por meio de microscópios, da coloração de Gram e das tiras impregnadas com reagentes conhecidas como dipsticks [Reine and Langston 2005, Bolodeoku and Donaldson 1996].

A tira é constituída por um suporte plástico contendo áreas impregnadas com reagentes químicos, conforme mostra a Figura 1. Quando as áreas de química seca entram em contato com a urina, uma reação de cor se desenvolve a qual permite a interpretação quase que imediata desses parâmetros químicos [Lima et al. 1992]. Os onze parâmetros analisados pela fita reagente da marca Uriscan são ácido ascórbico, bilirrubina, cetonas, densidade, glicose, leucócitos, nitrito, pH, proteínas, sangue e urobilinogênio.

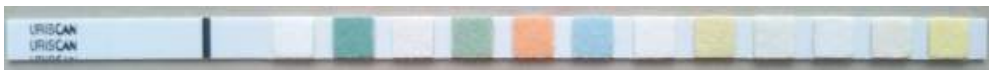

Figura 1. Fita reagente da marca Uriscan.

Existem no mercado instrumentos que executam a leitura das fitas reagentes, eliminando a subjetividade do olho humano na leitura das mudanças de cor, melhorando assim o grau de precisão [Ravel 1995]. Apesar dos avanços tecnológicos ocorridos nos últimos anos, e da análise das fitas reagentes tornarem o diagnóstico mais rápido, simples e econômico, este processo ainda peca em alguns pontos. Os laboratórios possuem apenas duas alternativas quando o assunto é a análise dos dipsticks. A primeira opção é realizar a leitura das fitas manualmente por meio da análise visual, comparando a amostra com uma tabela de cores padrão. A segunda alternativa é utilizar equipamentos especializados para a análise. Porém, devido ao custo destes equipamentos, muitos laboratórios ficam restritos apenas à análise manual realizada pelos profissionais, um processo sujeito a erros de diagnóstico devido à subjetividade da interpretação humana.

$\mathrm{Na}$ área de Visão Computacional o processo de extração de características fornece informações para a etapa de classificação. Várias características podem ser obtidas de objetos, tais como estatísticas, histogramas, forma, tamanho, textura e frequência [de Queiroz and Gomes 2006]. Quanto mais discriminantes forem essas informações, mais eficiente será o processo de classificação e melhor será a tomada de decisão do classificador. Vários trabalhos como [Filho et al. 2014] e [Hanusiak et al. 2010] mostram que combinações de características podem ser complementares, auxiliando, desta forma, na tomada de decisão dos classificadores. 
Neste trabalho é apresentada uma proposta que visa analisar o uso de técnicas de Visão Computacional para a classificação dos analitos presentes nos dipsticks, auxiliando, desta forma, a análise de urina e, posteriormente, incorporando um novo equipamento de baixo custo na rotina dos laboratórios.

\section{Análise de Urina}

$\mathrm{Na}$ literatura existente não foi encontrado nenhum trabalho relacionado ao aqui apresentado, porém algumas características presentes nos equipamentos de análise de urina são apresentados.

Um analisador de urina é um dispositivo utilizado no cenário clínico para realizar testes de modo automatizado. Esses equipamentos podem detectar e quantificar um número de analitos incluindo bilirrubina, proteína, glicose e sangue. Muitos modelos realizam a análise a partir de leitores de tiras de urina, um tipo de fotômetro de reflectância que pode processar várias centenas de tiras por hora. A reflectância se dá na relação da luminosidade refletida pela fita e o fluxo luminoso que incide sobre ela, fornecendo as informações necessárias para obtenção dos resultados de cada analito.

Atualmente, existem no mercado diversos equipamentos que permitem a análise dos dipsticks. Todos tem como objetivo otimizar o fluxo de trabalho, gerenciando as análises de forma eficiente e fornecendo resultados confiáveis para detectar estágios iniciais de muitas doenças, como infecções do trato urinário, doenças renais ou diabetes. Além disso, o uso do dispositivo elimina a subjetividade da avaliação visual da tira e minimiza os riscos associados à transcrição manual dos resultados. É o caso do equipamento Uriscan Pro II (Figura 2).

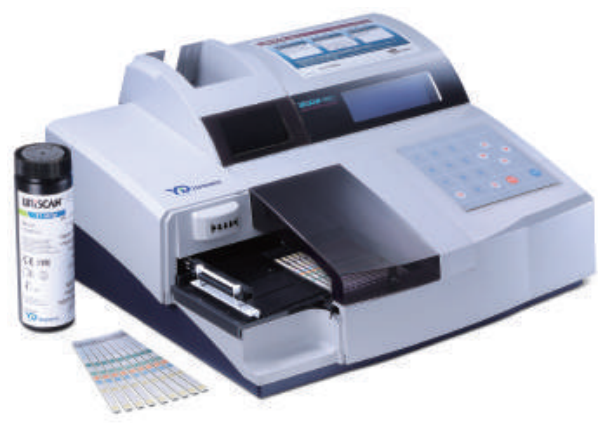

Figura 2. Equipamento Uriscan Pro II.

O analisador Uriscan Pro II pode ser utilizado em locais onde a demanda é média, com capacidade de análise de 720 tiras de teste por hora. O equipamento é suportado por um leitor de código de barras e uma impressora térmica [YD Diagnostics 2016]. Além da interface com código de barras, o equipamento também possui interface com o computador, mas é lenta e não prática, segundo informações do laboratório no qual foi desenvolvido o presente trabalho.

\section{Materiais e Métodos}

Para a aquisição das imagens, conforme a proposta do presente trabalho, utilizou-se o scanner HP Scanjet 3800 Photo. Com o intuito de facilitar a detecção da localização da 
fita, foi desenvolvida uma grade de alumínio de tamanho $30 \times 22 \mathrm{~cm}$ contendo espaço para o posicionamento de 10 fitas. Cada um desses espaços possui tamanho $0,55 \mathrm{x} 12,55$ $\mathrm{cm}$ e espaçamento de $2 \mathrm{~cm}$ entre as mesmas (Figura 3 ).

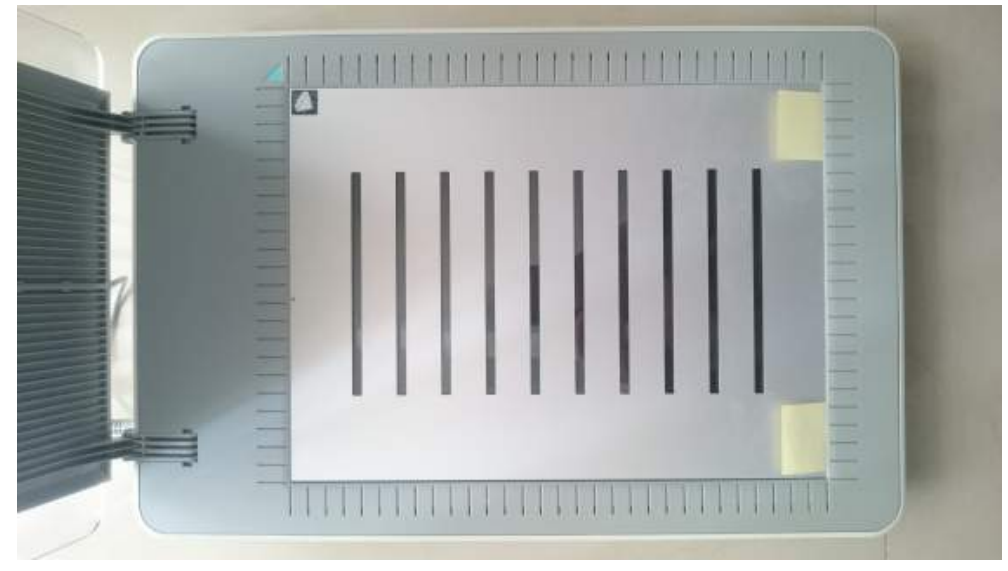

Figura 3. Grade posicionada no scanner.

As 300 urinas utilizadas para os testes estavam conservadas em geladeira no laboratório. As mesmas ficam armazenadas por uma semana e são descartadas após esse período.

O primeiro passo para que as análises pudessem ser feitas foi realizar as alíquotas das urinas que passariam pelo processo. Para tal, transferiu-se uma pequena quantidade para tubos de ensaio de fundo cônico, conforme Figura 4.

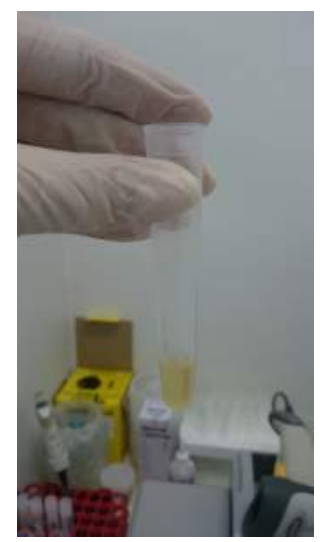

Figura 4. Alíquota de urina.

Após as urinas serem alíquotadas e registradas na máquina Uriscan Pro II, para cada urina, uma fita foi submergida e posicionada no equipamento. O segundo passo foi mergulhar novas fitas nas mesmas urinas e posicioná-las no scanner. No momento da aquisição da imagem, a resolução selecionada foi de 300 dpi.

Apesar da grade desenvolvida ter espaços para dez fitas reagentes, foram feitas imagens de 5 em 5 dipsticks, conforme a Figura 5, para que o tempo das primeiras fitas posicionadas não extrapolassem o tempo ideal de leitura, que fica entre um e dois minutos, 
segundo a bula. Além disso, para que todas as fitas estivessem no seu tempo ótimo de análise, aguardou-se um minuto após o posicionamento da última no scanner.

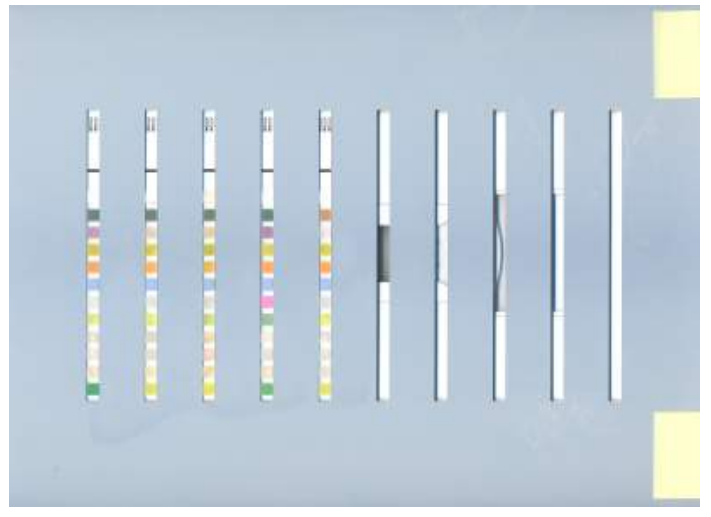

Figura 5. Exemplo de imagem adquirida.

A partir das imagens adquiridas, o primeiro passo do algoritmo realiza a segmentação das fitas. As coordenadas $X$ e $Y$, inferior e superior, de cada dipstick da grade foram obtidas manualmente e implementadas internamente no algoritmo. $\mathrm{O}$ segundo passo consiste em segmentar as áreas reagentes de cada uma das fitas. Assim como no passo anterior, as coordenadas de cada área foram obtidas manualmente, e seus valores utilizados no algoritmo. Essas áreas foram segmentadas de modo a obter-se uma imagem de 50x50 pixels para adição na base de dados e posterior extração de informações.

\subsection{Criação da Base Patterns_1}

Para a criação da base, cada padrão ou área reagente, de cada fita, obtido pelas imagens escaneadas e segmentadas pelo algoritmo, foram salvas com os valores impressos pelo equipamento. Logo, as imagens foram nomeadas de acordo com o padrão que a mesma representava. Essas foram organizadas em onze pastas, onde cada uma delas representa um item analisado pela fita. Em cada uma desses onze diretórios encontram-se 300 imagens, de tamanho 50X50 pixels totalizando 3300 imagens.

Porém, em alguns casos, os padrões extremos de alguns analitos não foram obtidos, e, para valores próximos desses extremos poucas imagens foram adquiridas, tendo em vista a sua dificuldade de obtenção.

\subsection{Extração de informações}

A extração de informações das imagens organizadas na base de dados Patterns_1 seguiu os passos:

- Converter a imagem para o modelo de cores HSV;

- Encontrar os valores máximo e mínimo para cada analito em cada canal HSV;

- Calcular para cada imagem a média dos valores dos canais H, S e V;

- Normalizar linearmente as médias dos canais, para ajustar as escalas de valores dos atributos para o intervalo $[0,1]$. 
A normalização seguiu a equação 1:

$$
f(x)=\frac{\bar{X}-\text { Min }}{\text { Max }- \text { Min }}
$$

Onde $\bar{X}$ é o valor a ser normalizado e $\operatorname{Max}$ e $\operatorname{Min}$ são os valores máximos e mínimos para cada canal de cor.

Visando ter informações complementares no modelo HSV, optou-se por utilizar, além das médias de cada canal, os valores de máximo e mínimo normalizados dos canais $\mathrm{H}, \mathrm{S}$ e V. Alternativamente, a mesma abordagem foi tomada seguindo o modelo RGB.

\section{Resultados e Discussão}

Os resultados apresentados a seguir foram obtidos através da conversão das imagens da base Patterns_1 para o modelo de cores HSV e dos atributos sendo representados pelas médias normalizadas de cada canal. Conforme descrito, optou-se por utilizar também o modelo de cores RGB e HSV com os valores normalizados de máximo e mínimo de cada canal como atributo, aliados as médias normalizadas. Porém, para os testes realizados com essas duas outras abordagens descritas não foram obtidos resultados significativos, comparados com os apresentados pela primeira abordagem, pois o uso desses valores não trouxe mudanças expressivas em relação a separação dos dados. Isso porque não foram obtidas informações adicionais que pudessem discriminar melhor as classes, resultando em uma projeção dos dados similar as obtidas com a utilização das médias normalizadas de cada canal $\mathrm{H}$, S e V.

Os testes foram realizados através da técnica de cross-validation, denominado $k$-fold. Nesse procedimento o conjunto de dados é dividido em $k$ conjuntos menores (folds), e para cada um desses $k$ conjuntos, um modelo é treinado utilizando os outros $k$ - 1 conjuntos como base de treinamento. No fim do processo, a taxa de acerto obtida pelo classificador é dada pela média dos valores provenientes de cada modelo obtido. No presente trabalho, optou-se por utilizar 10 folds.

Selecionada a opção de como os testes seriam executados, o próximo passo foi avaliar a performance do classificador de Redes Neurais (Multilayer Perceptron), a partir da manipulação do número de camadas e sua quantidade de neurônios, da taxa de aprendizado, momento e ciclos de treinamento.

A primeira etapa foi variar o número de neurônios nas camadas escondidas. O padrão trazido pelo Weka é de uma camada escondida representado pela letra a. Para as $M L P S$ deste trabalho, utilizou-se além do valor padrão outros dois valores que definem a rede com duas e três camadas escondidas. Para a rede com duas camadas escondidas, a definição da quantidade de neurônios de cada camada deu-se através da combinação da quantidade de atributos e da quantidade de classes. Enquanto que no caso da rede de três camadas escondidas, a quantidade de neurônios foi definida a partir da combinação dos valores descritos anteriormente, mais a soma da quantidade de atributos e de classes. Com o intuito de facilitar na descrição dos resultados, as abordagens descritas foram denominadas de 2 Camadas e 3 Camadas. 
Tendo em vista que cada item avaliado da fita reagente possui um número de classes diferentes, na Tabela 1 seguem os valores utilizados para o número de neurônios de cada camada.

\begin{tabular}{lccc} 
& Atributos & Classes & Atributos + Classes \\
\hline Ascórbico & 3 & 4 & 7 \\
Bilirrubina & 3 & 3 & 6 \\
Sangue & 3 & 5 & 8 \\
Cetonas & 3 & 5 & 8 \\
Densidade & 3 & 6 & 9 \\
Glicose & 3 & 6 & 9 \\
Leucócitos & 3 & 5 & 8 \\
Nitrito & 3 & 2 & 5 \\
pH & 3 & 8 & 11 \\
Proteínas & 3 & 6 & 9 \\
Urobilinogênio & 3 & 4 & 7
\end{tabular}

Tabela 1. Quantidade de neurônios de cada camada.

Todas as configurações de camadas utilizaram, durante os testes, os valores default para a taxa de aprendizado $(0,3)$, momento $(0,2)$ e ciclos de treinamento ( 500).

Utilizando-se os melhores percentuais obtidos na variação do número de camadas e suas respectivas quantidades de neurônios, o passo seguinte foi variar a taxa de aprendizado. Os valores utilizados foram $0,1,0,3,0,5,0,7,0,9$ e 1,0. Com a variação da taxa de aprendizado, foi possível observar que em alguns casos as taxas de acerto obtiveram uma pequena melhora, quando em comparação ao valor padrão $(0,3)$. No caso da densidade a melhora foi de $2 \%$, atingindo $74 \%$ com valor de aprendizado 0,1 . A glicose e os leucócitos apresentaram melhoria de 1\%, atingindo $99,67 \%$ e $88,67 \%$ respectivamente. Esses percentuais foram alcançados com os valores de taxa 0,7 e 1,0 para a glicose e 0, 5 e 1, 0 para os leucócitos. Para o sangue (95,33\%), nitrito $(99,33 \%)$, pH $(72,33 \%)$ e proteínas $(86,33 \%)$, as melhores taxas de acerto foram obtidas com o valor default de aprendizado $(0,3)$. $\mathrm{O}$ analito ascórbico apresentou $90,33 \%$ para aprendizado igual a 0,3 e 0,7 , enquanto que para as cetonas, com 95,33\%, as taxas de aprendizado foram 0,3 e 1,0. O urobilinogênio apresentou a mesma taxa de acerto para quase todos os valores de aprendizado, 98,33\% para 0,3, 0,5, 0,9 e 1,0. Já no caso da bilirrubina (99\%), o percentual foi o mesmo para todas as variações da taxa de aprendizado.

A partir das taxas de aprendizado definidas para cada analito, o próximo passo foi a variação do parâmetro momento, com os valores $0,1,0,2,0,3,0,5,0,7$ e 0,9 . Com a manipulação do parâmetro momento, foi possível observar que em apenas quatro casos as taxas de acerto obtiveram uma melhora, quando em comparação ao valor padrão $(0,2)$. Foram os casos dos padrões bilirrubina, glicose, leucócitos e pH. Os itens bilirrubina, glicose e leucócitos obtiveram uma melhora de 0,33\%, atingindo 99,33\%, 100\% e 89\% respectivamente. Para a bilirrubina o valor do momento foi de 0,9 , para a glicose o percentual foi atingido com os parâmetros 0,7 e 0,9 e para os leucócitos o momento foi de 0,1 . Para o $\mathrm{pH}$, o momento igual a $0,1,0,5$ e 0,7 proporcionou uma melhora de $1 \%$, atingindo uma taxa de acerto de $73,33 \%$.

O último passo dos testes com o algoritmo $M L P$ foi variar o parâmetro epoch ou 
ciclos de treinamento, com os valores $250,500,1000$ e 2000 . Foram utilizados a quantidade de camadas, neurônios, taxa de aprendizado e o momento, definidos nos passos anteriores para cada um dos analitos. A partir dos percentuais obtidos, foi possível perceber ganhos sutis para alguns dos itens analisados, quando comparados as taxa obtidas pelo valor padrão (500). É o caso da bilirrubina, densidade, leucócitos, pH e do urobilinogênio, atingindo $99,67 \%, 75 \%, 89,33 \%, 74 \%$ e $98,67 \%$, respectivamente. Nos casos da densidade, $\mathrm{pH}$ e do urobilinogênio essas taxas foram atingidas com o valor de ciclos igual a 1000. Para a bilirrubina, a melhor taxa foi obtida com o valor 1000 e 2000, enquanto que para os leucócitos foi o valor 250.

A Tabela 2 apresenta as melhores configurações da rede neural para cada analito. A partir dos valores obtidos para cada um dos parâmetros manipulados é notável que em sua maioria foram mantidos os valores padrões definidos pela ferramenta Weka. Avaliando-se o número de camadas e de neurônios a maioria dos analitos obtiveram melhores resultados com o valor a, que apresenta apenas uma camada e com o número de neurônios igual ao chão da média aritmética entre a quantidade de atributos e a quantidade de classes. No caso da taxa de aprendizado, oito dos onze itens apresentaram melhores percentuais com o valor 0,3 e apesar de algumas variações, o momento também mostrouse melhor com o valor default $(0,2)$. Para o parâmetro ciclos de aprendizado seis dos onze analitos atingiram os melhores percentuais por meio do valor padrão (500), quatro dos itens obtiveram os melhores percentuais com ciclos igual a 1000 e apenas um com o valor 250 .

\begin{tabular}{lccccc} 
& \# Camadas & Taxa de Aprendizado & Momento & Ciclos & Percentual \\
\hline Ascórbico & 2 Camadas & 0,3 & 0,2 & 500 & $90,33 \%$ \\
Bilirrubina & a & 0,3 & 0,9 & 1000 & $99,67 \%$ \\
Cetonas & $\mathrm{a}$ & 0,3 & 0,2 & 500 & $95,33 \%$ \\
Densidade & $\mathrm{a}$ & 0,1 & 0,2 & 1000 & $75 \%$ \\
Glicose & $\mathrm{a}$ & 0,7 & 0,7 & 500 & $100 \%$ \\
Leucócitos & 3 Camadas & 0,5 & 0,1 & 250 & $89,33 \%$ \\
Nitrito & 3 Camadas & 0,3 & 0,2 & 500 & $99,33 \%$ \\
pH & a & 0,3 & 0,1 & 1000 & $74 \%$ \\
Proteínas & 2 Camadas & 0,3 & 0,2 & 500 & $86,33 \%$ \\
Sangue & 3 Camadas & 0,3 & 0,2 & 500 & $95,33 \%$ \\
Urobilinogênio & a & 0,3 & 0,2 & 1000 & $98,67 \%$
\end{tabular}

Tabela 2. Parâmetros do MLP que obtiveram os melhores percentuais.

Dos onze itens analisados, os dois que apresentaram os menores percentuais foram a densidade e o $\mathrm{pH}$ que atingiram respectivamente $75 \%$ e $74 \%$. Tais resultados podem ser justificados pelas projeções dos dados de cada um dos analitos conforme a Figura 6 e a Figura 7.

É possível notar que nos casos da densidade e do pH há uma nuvem de exemplos de diferentes classes condensados, tornando a separação das amostras mais difícil. No caso da densidade, as classes 1015, 1020, 1025 e 1030 são as que se encontram mais embaraçadas, no canto superior esquerdo do gráfico, enquanto que para o analito $\mathrm{pH}$, os valores $5,5,5$ e 6 são os que apresentam maior proximidade. 


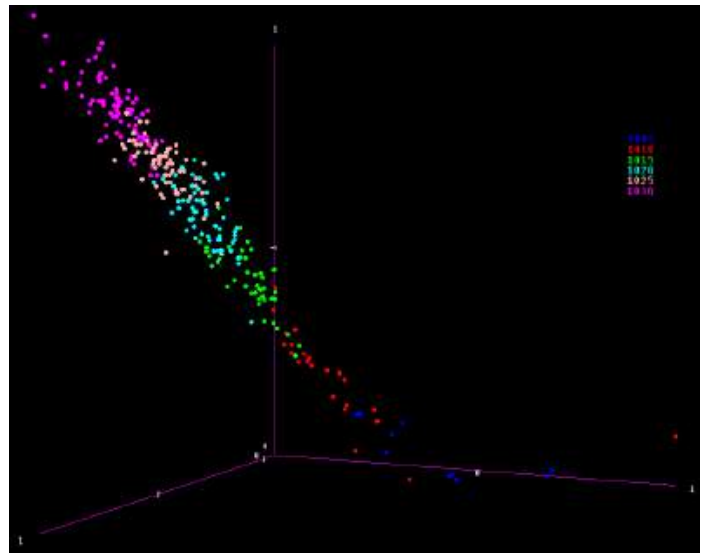

Figura 6. Projeção dos dados do analito densidade.

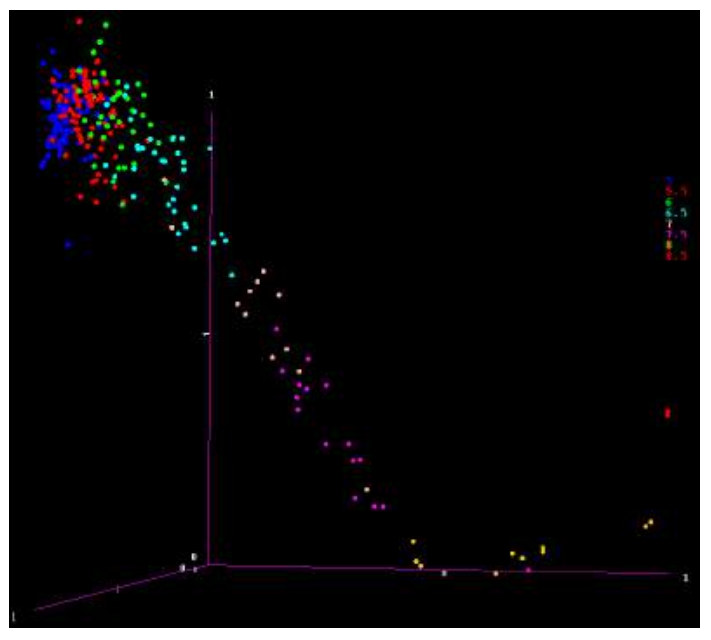

Figura 7. Projeção dos dados do analito pH.

\section{Conclusão}

No presente trabalho é proposta uma metodologia para análise das fitas reagentes no exame de urina utilizando-se um scanner de mesa convencional, técnicas de processamento de imagens e de aprendizado de máquina. Primeiramente construiu-se uma base de dados com imagens, adquiridas via scanner, de cada um dos itens analisados pela fita reagente. A partir destas imagens, foram extraídas informações que pudessem auxiliar no processo de classificação dos analitos.

A abordagem da extração de informações das imagens organizadas na base de imagens Patterns_1 foi feita por meio da conversão das imagens para o modelo de cores HSV e da obtenção dos valores médios normalizados de cada um dos canais.

O classificador $M L P$ foi testado através da manipulação do número de camadas e sua quantidade de neurônios, da taxa de aprendizado, momento e ciclos de treinamento. Em relação as configurações finais de cada analito, a maioria das MLPs obteve uma melhor resposta com os valores padrões definidos pela ferramenta Weka. No caso do número de camadas e de neurônios a maioria dos analitos obtiveram melhores resultados com o valor a. No caso da taxa de aprendizado, oito dos onze itens apresentaram melhores percentuais com o valor 0,3 e apesar de algumas variações, o momento também mostrou-se 
melhor com o valor default $(0,2)$. Para o parâmetro ciclos de aprendizado seis dos onze analitos atingiram os melhores percentuais por meio do valor padrão (500).

O MLP mostrou-se eficiente na classificação dos dados, obtendo $91,21 \%$ de taxa de acerto médio entre todos os analitos.

Apesar dos resultados promissores, é necessário aprimorar os métodos de extração e combinação de características, realizar testes com outros algoritmos de classificação e obter mais amostras dos casos extremos de cada padrão, que possam auxiliar na melhor definição das funções dos classificadores. Além disso, um estudo e avaliação das fitas reagentes faz-se necessário, medindo a variação das respostas entre fitas coletadas a partir de uma mesma amostra.

\section{Referências}

ABNT (2005). Associação Brasileira de Normas Técnicas. Laboratório clínico. Requisitos e recomendações para o exame da urina.

Amorim, A. E., Pacheco, J. B. P., Fernandes, T. T., and Biomedicina, F.-A. (2009). Exame de urina tipo i: freqüência percentual de amostras que sugerem infecção urinária. Anuário da Produção de Iniciação Científica Discente. 2008; 24 (12): 57, 68.

Bolodeoku, J. and Donaldson, D. (1996). Urinalysis in clinical diagnosis. Journal of clinical pathology, 49(8):623.

Chien, T.-I., Kao, J.-T., Liu, H.-L., Lin, P.-C., Hong, J.-S., Hsieh, H.-P., and Chien, M.-J. (2007). Urine sediment examination: a comparison of automated urinalysis systems and manual microscopy. Clinica Chimica Acta, 384(1):28-34.

de Queiroz, J. E. R. and Gomes, H. M. (2006). Introdução ao processamento digital de imagens. RITA, 13(2):11-42.

Filho, P. L. P., Oliveira, L. S., Nisgoski, S., and Britto Jr, A. S. (2014). Forest species recognition using macroscopic images. Machine Vision and Applications.

Hanusiak, R. K., Justino, E. J. R., Oliveira, L. S., and Sabourin, R. (2010). Identificação da autoria de manuscritos com base em atributos genéticos e genéricos da escrita. Revista de Informática Teórica e Aplicada, 17(2):193-209.

Lima, A. O., Soares, J. B., Greco, J., Galizzi, J., and Cançado, J. R. (1992). Métodos de laboratório aplicados à clínica: técnica e interpretaçäo. Guanabara Koogan.

Ravel, R. (1995). Laboratório clínico: aplicações clínicas dos dados laboratoriais. Guanabara Koogan.

Reine, N. J. and Langston, C. E. (2005). Urinalysis interpretation: how to squeeze out the maximum information from a small sample. Clinical techniques in small animal practice, 20(1):2-10.

Strasinger, S. (2000). Uroanálise e fluidos biológicos.

Strasinger, S. K. and Torquettitoloi, M. R. (1996). Uroanálise e fluidos biológicos. Editorial Premier.

YD Diagnostics, Y. (2016). Urican pro ii analyzer. http://www . yd-diagnostics.com/new/product/uriscan_3.php. Acessado em $15 / 08 / 2016$. 